\title{
COMUNICAÇÃO
}

\section{PREDIÇÃO DE MÉDIAS DE LINHAGENS OBTIDAS DE HIIBRIDOS SIMPLES DE MILHO (Zea mays L.)}

\author{
Prediction of maize (Zea mays L.) inbred lines means from single-crosses
}

Laryssa Ferreira Viana1, João Cândido de Souza ${ }^{2}$, Juarez Campolina Machado ${ }^{3}$, José Luiz Lima ${ }^{4}$

\begin{abstract}
RESUMO
A utilização de milho híbrido foi o principal fator responsável pelo aumento na produtividade de grãos. Para a obtenção de híbridos é necessária a identificação da população mais promissora para a extração de linhagens. Entre os procedimentos que auxiliam os melhoristas nessa escolha, destaca-se a estimativa de $\mathrm{m}+\mathrm{a}$ ', que corresponde à média de todas as linhagens na geração S".O presente trabalho foi conduzido com os objetivos de estimar a contribuição dos locos em heterozigose (d) e a contribuição dos locos em homozigose $\left(\mathrm{m}+\mathrm{a}\right.$ ') de caracteres morfofisiológicos para verificar o potencial de híbridos comerciais. Foram utilizadas as gerações $\mathrm{F}_{1}$ e $\mathrm{F}_{2}$ de dez híbridos simples comerciais. Os experimentos foram conduzidos em Lavras, na Fazenda Experimental Vitorinha e em Ijaci, no ano agrícola de 2005/06. Os tratamentos foram avaliados utilizando-se o delineamento em blocos casualizados, com três repetições, sendo os dados tomados em cinco plantas/repetição. Os caracteres avaliados foram: altura de plantas, altura de espiga, número de folhas acima da espiga, diâmetro do colmo, comprimento da espiga, diâmetro da espiga, diâmetro do sabugo, peso da espiga e peso de grãos. Maiores estimativas de m+a' para o caráter peso de grãos foram obtidas para os híbridos A2555 e AG7000, evidenciando o maior potencial para extração de linhagens. A heterose foi responsável, em média, por $69,36 \%$ da produção da geração $F_{1}$ As correlações entre o desempenho da geração $\mathrm{F}_{1}$ e m+a' e $\mathrm{F}_{1}$ e d foram não significativas indicando não haver possibilidade de selecionar populações com base na média da geração $\mathrm{F}_{1}$.
\end{abstract}

Termos para indexação: Milho, estimativas de m+a'e d, extração de linhagens.

\begin{abstract}
The use of hybrid corn was the main factor in grain yield increase. In order to obtain hybrids, the identification of the best population for inbred lines extraction is needed. The m+a'estimation is an outstanding procedure for such a choice. It stands for the means of all inbred lines in the generation S". This work was carried out with the aim of estimating the contribution of both locus in heterozigosis (d) and locus in homozigosis $(\mathrm{m}+\mathrm{a})$ ) of morphophisiological characters in order to find out the potential of commercial hybrids. Both $F_{1}$ and $F_{2}$ generations of ten commercial ordinary hybrids were used. The experiments were carried out in Lavras, at Fazenda Experimental Vitorinha and in Ijaci in the agricultural year of 2005/06. The treatments were evaluated by using a randomized complete block design with three replications. Data were taken from five plants. The evaluated characteristics were as it follows: plant height, ear height, number of leaves over the ear, colm diameter, ear length, ear diameter, corncob length , ear weight, and grain yield. Higher m+a' estimates for grain yield were reached for both A2555 and AG7000 hybrids, thus showing the best potential for inbred lines extractions. The heterosis was found to account for $69,36 \%$ of the $\mathrm{F}_{1}$ generation production. The correlations between the $\mathrm{F}_{1}$ generation performance and $\mathrm{m}+\mathrm{a}$, and between $\mathrm{F}_{1}$ and $\mathrm{d}$ were not significant showing that the population selection of $\mathrm{F}_{1}$ generation means may not be taken as a ground for selecting populations.
\end{abstract}

Index terms: Corn, $\mathrm{m}+\mathrm{a}$ ' and $\mathrm{d}$,inbred lines extraction.

(Recebido em 4 de setembro de 2007 e aprovado em 10 de junho de 2008)

O sucesso de um programa de melhoramento de milho visando à obtenção de híbridos está ligado à identificação da população mais promissora para a extração de linhagens. Além disso, devem-se associar as estimativas de capacidade de combinação da população com a estimativa de $\mathrm{m}+\mathrm{a}$ ' da mesma. Entre os procedimentos que auxiliam os melhoristas nessa escolha, destaca-se a estimativa de m+a', que corresponde à média de todas as linhagens na geração S”. Esse método proposto por Vencovsky (1987) permite avaliar o potencial das populações em gerações precoces, obtendo a estimativa por meio do contraste entre as gerações $\mathrm{F}_{1}$ e $\mathrm{F}_{2}$.

O desempenho da geração $\mathrm{F}_{1}$ de um híbrido é função de $m+a^{\prime}+d$, em que $m+a^{\prime}$ é a contribuição dos locos

${ }^{1}$ Mestre em genética e melhoramento de plantas - UFLA-MG - email: laryssa.viana@bol.com.br

2Professor do Departamento de Biologia UFLA-MG - email: cansouza@ufla.br

${ }^{3}$ Mestre em genética e melhoramento de plantas - UFLA-MG email: juarezcmachado@yahoo.com.br

${ }^{4}$ Mestre em genética e melhoramento de plantas - UFLA-MG email:lima_|@bol.com.br 
em homozigose, fixados nas linhagens parentais, e d é o desvio dos heterozigotos em relação à média. Assim, m+a' depende do desempenho das linhagens per se e d depende da divergência entre as linhagens e da existência de dominância no controle do caráter. Do exposto, para se obter bons híbridos, é necessário bom desempenho das linhagens per se e que elas sejam divergentes. O procedimento tem sido utilizado no Brasil, em algumas oportunidades (Vianna et al., 1982; Lima et al., 1984; Teresawa Júnior, 1993; Nass \& Miranda Filho, 1995; Packer, 1998; Souza Sobrinho, 2001), quando se constatou variação entre as populações.

Várias são as opções de populações que podem ser utilizadas para a extração de linhagens para a produção de híbridos. A mais viável, no entanto, parece ser a utilização de híbridos simples comerciais, pois têm a vantagem de já terem sido testados em vários ambientes, associando, dessa forma, alta produtividade com grande proporção de locos favoráveis fixados. Além disso, são altamente heterozigóticos, associando, desse modo, média alta e grande variação.

O método, frequentemente utilizado para obtenção de linhagens homozigóticas, é a autofecundação artificial, tendo como consequência principal a depressão por endogamia. Assim, o valor de uma população como fonte de linhagens é dependente da depressão por endogamia em relação às várias características, principalmente rendimento de grãos, o que limita a obtenção de boas linhagens para a posterior obtenção de híbridos vigorosos (Lima et al., 1984). Existem vários trabalhos que citam as estimativas de depressão por endogamia para várias características, empregando diferentes populações em milho (Hallauer \& Sears, 1973; Good \& Hallauer, 1977; Vianna et al., 1982; Lima et al., 1984; Nass \& Miranda Filho, 1995; Vasal et al., 1995). No contexto de caracteres morfofisiológicos, Farias Neto \& Miranda Filho (2000) identificaram expressiva depressão por endogamia para altura de plantas e espigas utilizando duas populações de milho.

Diante do exposto, neste trabalho, objetivou-se estimar a contribuição dos locos em heterozigose (d) e a contribuição dos locos em homozigose $(m+a)$ de caracteres morfofisiológicos como indicadores do potencial de híbrido de milho para extração de linhagens em programas de melhoramento.

Para a realização desse trabalho foram utilizadas as gerações $\mathrm{F}_{1}$ e $\mathrm{F}_{2}$ de dez híbridos simples comerciais (Tabela 1). De posse da geração $F_{1}$ desses híbridos, adquiridas no comércio local, foram obtidas as gerações $\mathrm{F}_{2}=\mathrm{S}_{0}$ de cada híbrido, por meio de autofecundações controladas no campo experimental do Departamento de Biologia da Universidade Federal de Lavras (DBI/UFLA).

Os experimentos foram conduzidos em Lavras na Área Experimental DBI/UFLA e na Fazenda Experimental Vitorinha, e em Ijaci na Fazenda Palmital, no ano agrícola de 2005/06. Os experimentos da geração $F_{1}$ e $F_{2}$ foram contíguos, conduzidos em delineamento de blocos casualizados com três repetições, sendo os dados tomados de cinco plantas aleatórias na parcela/repetição. As parcelas foram constituídas por duas linhas de quatro metros espaçadas de $50 \mathrm{~cm}$. Os tratos culturais foram os recomendados para a cultura do milho na região sul de Minas Gerais. Os caracteres avaliados foram: altura de plantas, altura de espiga, número de folhas acima da espiga, diâmetro do colmo, comprimento da espiga, diâmetro da espiga, diâmetro do sabugo, peso da espiga e peso de grãos utilizando uma amostra de cinco plantas na parcela.

Foi realizada uma análise de variância por local e, posteriormente, a análise conjunta dos dados segundo Ramalho et al. (2005). As análises de variância para todas

Tabela 1 - Descrição dos dez híbridos simples comerciais:

\begin{tabular}{ccccc}
\hline Híbrido & Nome & Empresa & Textura dos grãos & Ciclo $^{1 /}$ \\
\hline 1 & DKB 199 & Monsanto & Semiduro & SMP \\
2 & $30 F 90$ & Pioneer & Duro & SMP \\
3 & A2555 & Nidera Sementes & Duro & SMP \\
4 & DKB333B & Monsanto & Semiduro & SMP \\
5 & DOW657 & Dow AgroScience & Semiduro & P \\
6 & AG8060 & Monsanto & Semiduro & P \\
7 & $30 F 87$ & Pioneer & Duro & SMP \\
8 & DOW8420 & Dow AgroScience & Duro & P \\
9 & $30 K 75$ & Pioneer & Semiduro & SMP \\
10 & AG7000 & Monsanto & Semiduro & SMP \\
\hline
\end{tabular}

${ }^{1 /} \mathrm{P}$ - ciclo precoce; SMP- ciclo semiprecoce 
as características foram realizadas no PROC GLM do SAS (SAS Institute, 2000). A partir das médias das gerações avaliadas, foram obtidas estimativas da contribuição dos locos em homozigose $(m+a)$ e estimativas da contribuição dos locos em heterozigose (d). Considerando $\mathrm{F}_{1}=\mathrm{m}+\mathrm{a}+\mathrm{d}$ e $\mathrm{F}_{2}=\mathrm{m}+\mathrm{a}+(1 / 2) \mathrm{d}$, o contraste $2 \mathrm{~F}_{2}-\mathrm{F}_{1}$ fornece a estimativa de $\mathrm{m}+\mathrm{a}$ ' e $2\left(\mathrm{~F}_{1}-\mathrm{F}_{2}\right)$ fornece a estimativa de $\mathrm{d}$.

Foram estimadas as correlações fenotípicas entre o desempenho da geração $F_{1}$ dos híbridos e a estimativa de $\mathrm{m}+\mathrm{a}$ ' e correlações entre o desempenho da geração $\mathrm{F}_{1}$ e a estimativa de d para os caracteres morfofisiológicos baseados na análise conjunta dos dados fornecidos pela expressão: $\operatorname{rxy}=\mathrm{COVxy/ \sigma x} . \sigma \mathrm{y}$

$\mathrm{Na}$ análise de variância conjunta, envolvendo os três locais, verificou-se que a precisão experimental, avaliada pelo coeficiente de variação, foi abaixo de $18,3 \%$ para a geração $F_{1}$, sendo considerado um bom valor para a cultura do milho. $\mathrm{Na}$ geração $\mathrm{F}_{2}$, o valor foi abaixo de $30,51 \%$, sendo considerado médio (Tabelas 2 e 3 ).

Tabela 2 - Resumo da análise de variância para altura de planta (AP), altura de espiga (AE), número de folhas acima da espiga ( $n^{\circ}$ f.ac.esp.), diâmetro de colmo ( $\varnothing$ colmo), comprimento de espiga (com.espiga), diâmetro de espiga ( $\varnothing$ espiga), diâmetro de sabugo ( $\varnothing$ sabugo), peso de espiga (PE) e peso de grãos (PG) para a geração $\mathrm{F}_{1}$ de híbridos simples de milho. UFLA, Lavras, MG, safra 2005/2006.

\begin{tabular}{|c|c|c|c|c|c|}
\hline $\mathrm{FV}$ & C.V. (\%) & Média & Trat. & Local & Local*trat. \\
\hline G.L. & & & 9 & 2 & 18 \\
\hline $\mathrm{AP}(\mathrm{m})$ & 5,51 & 2,2 & $0,80 * *$ & $29,78 * *$ & $0,16^{* *}$ \\
\hline $\mathrm{AE}(\mathrm{m})$ & 9,25 & 1,19 & $0,14 * *$ & $9,86 * *$ & $0,07 * *$ \\
\hline $\mathrm{n}^{\circ}$ f.ac.esp. & 8,56 & 6,29 & $4,75 * *$ & $16,08 * *$ & $0,49 *$ \\
\hline$\Phi \operatorname{colmo}(\mathrm{cm})$ & 15,06 & 1,93 & $0,63 * *$ & $16,75^{* *}$ & $0,28 * *$ \\
\hline com. Espiga $(\mathrm{cm})$ & 11,73 & 16,82 & $18,23 * *$ & $128,33 * *$ & $19,42 * *$ \\
\hline$\Phi$ espiga(cm) & 6,53 & 4,79 & $0,23 *$ & $15,44 * *$ & $0,19 * *$ \\
\hline$\Phi$ sabugo $(\mathrm{cm})$ & 6,79 & 2,72 & $0,72 * *$ & $1,21 * *$ & $0,16^{* *}$ \\
\hline $\mathrm{PE}(\mathrm{g})$ & 18,31 & 248,49 & $8043,54 * *$ & $726247,24 * *$ & $6015,54 * *$ \\
\hline PG(g) & 17,52 & 209,59 & $4608,30 * *$ & $487452,72 * *$ & $4340,44 * *$ \\
\hline
\end{tabular}

Tabela 3 - Resumo da análise de variância para altura de planta (AP), altura de espiga (AE), número de folhas acima da espiga ( $n^{\circ}$ f.ac.esp.), diâmetro de colmo ( $\varnothing$ colmo), comprimento de espiga (com.espiga), diâmetro de espiga ( $\varnothing$ espiga), diâmetro de sabugo ( $\varnothing$ sabugo), peso de espiga (PE) e peso de grãos (PG) para a geração $\mathrm{F}_{2}$ de híbridos simples de milho. UFLA, Lavras, MG, safra 2005/2006.

\begin{tabular}{cccccc}
\hline FV & C.V. $(\%)$ & Média & Trat. & Local & Local*trat. \\
\hline GL & & & 9 & 2 & 18 \\
AP(m) & 10,15 & 1,76 & $0,42^{* *}$ & $19,91^{* *}$ & $0,11^{* *}$ \\
AE(m) & 15,55 & 0,91 & $0,19 * *$ & $5,88^{* *}$ & $0,06 * *$ \\
$n^{\text {o }}$ f.ac.esp. & 10,45 & 6,07 & $2,34 * *$ & $16,60^{* *}$ & $0,57 \mathrm{n} . \mathrm{s}$. \\
$\Phi$ colmo $(\mathrm{cm})$ & 20,78 & 1,65 & $0,33^{* *}$ & $21,86^{* *}$ & $0,35^{* *}$ \\
com. Espiga $(\mathrm{cm})$ & 15,9 & 15,17 & $19,21^{* *}$ & $52,55^{* *}$ & $17,24^{* *}$ \\
$\Phi$ espiga $(\mathrm{cm})$ & 8,87 & 4,29 & $0,54 * *$ & $8,58^{* *}$ & $0,28^{* *}$ \\
$\Phi$ sabugo $(\mathrm{cm})$ & 9,11 & 2,58 & $1,10^{* *}$ & $0,56^{* *}$ & $0,10^{*}$ \\
PE $(\mathrm{g})$ & 29,78 & 163,56 & $7891,32^{* *}$ & $397046,54 * *$ & $4061,64 *$ \\
PG $(\mathrm{g})$ & 30,51 & 136,9 & $7068,84^{* *}$ & $278676,00^{* *}$ & $3106,15^{*}$ \\
\hline
\end{tabular}


Foi constatado, na análise de variância conjunta, que a fonte de variação tratamentos foi significativa pelo teste $\mathrm{F}(\mathrm{P}<0,01)$ para todos os caracteres, indicando que há diferença entre os híbridos. O mesmo pode ser observado para as fontes locais, indicando que os locais são diferentes entre si. A interação TxL também foi significativa, com exceção para o caráter número de folhas acima da espiga da geração $F_{2}$, indicando comportamento diferenciado relativo dos diferentes genótipos nos distintos ambientes (Tabelas 2 e 3 ).
As médias dos caracteres avaliados das gerações $\mathrm{F}_{1}$ e $\mathrm{F}_{2}$ dos híbridos são apresentadas nas Tabelas 4 e 5. Pode-se observar que as médias dos caracteres avaliados da geração $\mathrm{F}_{1}$ foram maiores que as médias da geração $F_{2}$. Embora não seja possível dizer se as diferenças são significativas, os caracteres peso de espiga e peso de grãos apresentaram diferenças de maiores amplitudes que os demais caracteres, provavelmente pelo fato de que o rendimento está relacionado com a herança do caractere (dominância/ heterose) do que ao maior efeito ambiental.

Tabela 4 - Médias da geração $\mathrm{F}_{1}$ para os caracteres altura de plantas (AP), altura de espiga (AE), número de folhas acima da espiga ( $n^{\circ}$ f.ac.esp.), diâmetro do colmo ( $\varnothing$ colmo), comprimento da espiga (Com.Espiga), diâmetro da espiga ( $\varnothing$ espiga), diâmetro do sabugo ( $\varnothing$ sabugo), peso espiga (PE), peso de grãos (PG) dos híbridos avaliados com base na análise conjunta. UFLA, Lavras, MG, safra 2005/2006.

\begin{tabular}{cccccccccc}
\hline HS & AP & AE & $n^{\text {o }}$ f.ac.esp. & $\varnothing$ colmo & $\begin{array}{c}\text { Com. } \\
\text { Espiga }\end{array}$ & $\emptyset$ espiga & $\varnothing$ sabugo & PE & PG \\
\hline 1 & 2,37 & 1,23 & 6,64 & 2,17 & 17,28 & 4,68 & 2,75 & 254,22 & 211,17 \\
2 & 2,43 & 1,28 & 6,57 & 2,01 & 17,32 & 4,78 & 2,62 & 255,22 & 218,42 \\
3 & 2,04 & 1,1 & 6,22 & 1,78 & 16,64 & 4,78 & 2,58 & 242,4 & 207,75 \\
4 & 2,16 & 1,13 & 6,48 & 2,05 & 16,67 & 4,76 & 2,66 & 253,17 & 211,06 \\
5 & 2,13 & 1,2 & 6,42 & 1,88 & 15,75 & 4,73 & 2,66 & 221,66 & 190,86 \\
6 & 2,33 & 1,19 & 6,71 & 1,93 & 18,11 & 4,9 & 2,92 & 272,2 & 227,53 \\
7 & 2,17 & 1,23 & 6,2 & 1,87 & 16,7 & 4,78 & 2,56 & 246,28 & 208,97 \\
8 & 2,06 & 1,14 & 5,95 & 1,81 & 16,62 & 4,92 & 2,91 & 253,93 & 210,71 \\
9 & 2,13 & 1,15 & 5,8 & 1,84 & 16,69 & 4,76 & 2,8 & 249,73 & 212,2 \\
10 & 2,14 & 1,2 & 5,91 & 1,9 & 16,33 & 4,78 & 2,7 & 236,08 & 197,15 \\
\hline Geral & 2,196 & 1,185 & 6,29 & 1,924 & 16,81 & 4,787 & 2,716 & 248,48 & 209,58 \\
\hline
\end{tabular}

Tabela 5 - Médias da geração $\mathrm{F}_{2}$ para os caracteres altura de plantas (AP), altura de espiga (AE), número de folhas acima da espiga ( $n^{\circ}$ f.ac.esp.), diâmetro do colmo ( $\varnothing$ colmo), comprimento da espiga (Com.Espiga), diâmetro da espiga ( $\varnothing$ espiga), diâmetro do sabugo ( $\varnothing$ sabugo), peso espiga (PE), peso de grãos (PG) dos híbridos avaliados com base na análise conjunta. UFLA, Lavras, MG, safra 2005/2006.

\begin{tabular}{cccccccccc}
\hline $\mathrm{F}_{2}$ & $\mathrm{AP}$ & $\mathrm{AE}$ & $\mathrm{n}^{\circ}$ f.ac.esp. & $\varnothing$ colmo & $\begin{array}{c}\text { Com. } \\
\text { Espiga }\end{array}$ & $\varnothing$ espiga & $\varnothing$ sabugo & PE & PG \\
\hline 1 & 1,79 & 0,89 & 6,35 & 1,71 & 15,53 & 4,25 & 2,61 & 169,33 & 140,37 \\
2 & 1,95 & 1,04 & 6,15 & 1,69 & 14,35 & 4,2 & 2,5 & 153,84 & 131,46 \\
3 & 1,72 & 0,86 & 6,02 & 1,75 & 15,77 & 4,27 & 2,54 & 172,73 & 156,6 \\
4 & 1,67 & 0,82 & 6,22 & 1,72 & 15,95 & 4,26 & 2,45 & 172,02 & 144,15 \\
5 & 1,74 & 0,95 & 6,26 & 1,74 & 15,6 & 4,22 & 2,5 & 156,2 & 130,75 \\
6 & 1,88 & 0,94 & 6,33 & 1,68 & 15,75 & 4,36 & 2,8 & 176,95 & 146,75 \\
7 & 1,75 & 0,95 & 5,97 & 1,56 & 14,16 & 4,2 & 2,29 & 149,31 & 125,42 \\
8 & 1,67 & 0,87 & 5,75 & 1,53 & 14,72 & 4,52 & 2,81 & 167,42 & 137,6 \\
9 & 1,67 & 0,84 & 5,86 & 1,53 & 14,6 & 4,18 & 2,64 & 138,91 & 112,06 \\
10 & 1,67 & 0,88 & 5,75 & 1,59 & 15,2 & 4,42 & 2,62 & 178,91 & 143,8 \\
Geral & 1,751 & 0,904 & 6,06 & 1,65 & 15,163 & 4,28 & 2,576 & 163,56 & 136,89 \\
\hline
\end{tabular}


Dentre os caracteres morfofisiológicos, maiores estimativas de $\mathrm{d}$, em média, foram obtidas para os caracteres peso de grãos $(69,3 \%)$, peso de espiga $(68,3 \%)$, altura de espiga $(47,4 \%)$ e altura de planta $(40,5 \%)$ (Tabela 6). Esses valores em porcentagem são obtidos pela razão da estimativa média de d e o desempenho da geração $F_{1}$ para o caractere em questão. Com base nesses valores, é esperado, por exemplo, que as plantas da geração $F_{2}$ apresentem redução em $20 \%$ na altura de plantas. Observase que o valor $20 \%$ representa a depressão por endogamia, isto é, é a metade do valor obtido pela contribuição dos locos em heterozigose (d) para o caractere altura de plantas. Esses resultados estão de acordo com relatos de Farias Neto \& Miranda Filho (2000) para a cultura do milho.

Pode-se observar que, em termos percentuais, a heterose foi responsável, na média dos dez híbridos avaliados, por $69,36 \%$ do peso de grãos da geração $F_{1}$, sendo os restantes $30,64 \%$ decorrentes da contribuição dos efeitos genéticos aditivos, herdados das linhagens parentais (Tabela 6). Estes resultados são concordantes com os apresentados por Souza Sobrinho (2001) que constatou que a estimativa média de $\mathrm{m}+\mathrm{a}$ ' representou $29,4 \%$ da média da geração $F_{1}$ dos híbridos e que, em média, o valor de d correspondeu a $70,4 \%$ da geração $F_{1}$ dos híbridos.

Tabela 6 - Estimativa média da contribuição dos locos em heterozigose (d), estimativa média dos locos em homozigose $\left(\mathrm{m}+\mathrm{a}\right.$ ') e as médias da geração $\mathrm{F}_{1}$ e $\mathrm{F}_{2}$ para os caracteres altura de plantas (AP), altura de espiga (AE), número de folhas acima da espiga ( ${ }^{\circ}$ f.ac.esp.), diâmetro de colmo ( $\varnothing$ colmo), comprimento de espiga (com.espiga), diâmetro de espiga ( $\varnothing$ espiga), diâmetro de sabugo ( $\varnothing$ sabugo), peso de espiga (PE) e peso de grãos (PG) para os caracteres avaliados. UFLA, Lavras, MG, safra 2005/2006.

\begin{tabular}{ccccc}
\hline Caracteres & $\mathrm{d}(\%)$ & $\mathrm{m}+\mathrm{a}^{`}(\%)$ & $\mathrm{F}_{1}$ & $\mathrm{~F}_{2}$ \\
\hline $\mathrm{AP}(\mathrm{m})$ & 40,53 & 59,65 & 2,196 & 1,751 \\
$\mathrm{AE}(\mathrm{m})$ & 47,43 & 53,16 & 1,185 & 0,904 \\
$\mathrm{n}^{\circ}$ f.ac.esp. & 7,12 & 93,00 & 6,29 & 6,06 \\
$\Phi$ colmo $(\mathrm{cm})$ & 28,48 & 71,73 & 1,924 & 1,65 \\
com. Espiga $(\mathrm{cm})$ & 19,61 & 80,43 & 16,81 & 15,163 \\
$\Phi$ espiga $(\mathrm{cm})$ & 20,85 & 79,17 & 4,787 & 4,28 \\
$\Phi$ sabugo $(\mathrm{cm})$ & 10,31 & 89,84 & 2,716 & 2,576 \\
PE $(\mathrm{g})$ & 68,36 & 31,65 & 248,48 & 163,56 \\
\hline $\mathrm{PG}(\mathrm{g})$ & 69,36 & 30,64 & 209,58 & 136,89 \\
\hline & & & &
\end{tabular}

Na tabela 7, pode-se observar que os híbridos 2, 7 e 9 foram os que apresentaram maiores estimativas de d, isto é, os híbridos que provavelmente apresentam maiores estimativas de heterose. Concordantemente, podemos observar que os híbridos 2, 7 e 9 foram os que apresentaram menores estimativas de m+a', isto é, os que apresentaram menor contribuição dos efeitos genéticos aditivos das linhagens parentais para o desempenho da geração $F_{1}$. Dentre estes, destacamos o híbrido 9 que apresenta $\mathrm{m}+\mathrm{a}$ ' de $5,6 \%$ e heterose de $94,4 \%$, ou seja, $94,4 \%$ da média da geração $F_{1}$ são decorrentes da heterose e apenas $5,6 \%$ decorrentes da média das linhagens parentais.

Tabela 7 - Estimativas da contribuição dos locos em heterozigose (d) e dos locos em homozigose (m+a') para o caractere peso de grãos dos híbridos. UFLA, Lavras, MG, safra 2005/2006.

\begin{tabular}{ccc}
\hline PG & $\mathrm{d}(\%)$ & $\mathrm{m}+\mathrm{a}(\%)$ \\
\hline 1 & 67,05 & 32,95 \\
2 & 79,63 & 20,38 \\
3 & 49,24 & 50,75 \\
4 & 63,40 & 36,60 \\
5 & 62,99 & 37,01 \\
6 & 71,01 & 29,00 \\
7 & 79,96 & 20,04 \\
8 & 69,39 & 30,61 \\
9 & 94,38 & 5,62 \\
10 & 54,12 & 45,87 \\
\hline
\end{tabular}

Além disso, pode-se observar que as maiores estimativas de $m+\mathrm{a}$ ' foram obtidas para os híbridos 3 e 10, evidenciando que eles apresentam o maior potencial para a extração de linhagens com maior produtividade de grãos; e o de menor potencial é o híbrido 9.

A estimativa de $m+a$ ' indica que se a população for utilizada para extração de linhagens, a média esperada das linhagens obtidas será igual a m+a'. Por outro lado, não se refere a capacidade de combinação das referidas linhagens. Dessa forma, seria interessante associar as estimativas de capacidade de combinação da população com a estimativa de $m+a$ ' da mesma. Usando esta combinação de informações, devem-se associar populações com melhores capacidades de combinação e melhores estimativas de $\mathrm{m}+\mathrm{a}$.

As correlações entre o desempenho das gerações $\mathrm{F}_{1}$ dos híbridos e m+a' e as correlações entre o desempenho 
das gerações $\mathrm{F}_{1}$ dos híbridos e $\mathrm{d}$ na maioria dos caracteres foram não significativas (dados não apresentados). Somente foram significativas as correlações entre o desempenho das gerações $\mathrm{F}_{1}$ dos híbridos e d para os caracteres número de folhas acima da espiga, diâmetro do colmo e comprimento da espiga e as correlações entre o desempenho das gerações $\mathrm{F}_{1}$ dos híbridos e $\mathrm{m}+\mathrm{a}$ ' para o caractere diâmetro do sabugo. Dessa forma, não há uma associação entre médias dos híbridos e médias das linhagens parentais, e entre médias dos híbridos e heterose. Isto significa que não é possível selecionar populações baseado apenas no desempenho da geração $\mathrm{F}_{1}$ do híbrido.

Pelo observado neste trabalho, pode-se concluir que as maiores estimativas de depressão por endogamia foram detectadas para peso de grãos, peso de espiga, altura de espiga e altura de planta. Os híbridos simples demonstram-se como boas alternativas para extração de linhagens. As correlações entre o desempenho da geração $\mathrm{F}_{1}$ e a estimativa de m+a' e entre o desempenho da geração $\mathrm{F}_{1}$ e d para os caracteres peso de espiga e peso de grãos foram não significativas. Assim, o desempenho do híbrido per se não é um bom parâmetro para a escolha de populações para extração de linhagens.

\section{REFERÊNCIAS BIBLIOGRÁFICAS}

FARIAS NETO, A.L. de; MIRANDA FILHO, J.B. Inbreeding in two maize subpopulations selected for tassel size. Scientia Agricola, Piracicaba, v.57, n.3, p.487490, 2000.

GOOD, R.L.; HALLAUER, A.R. Inbreeding depression in maize by selfing and full-sibbing. Crop Science, Madison, v.17, p.935-940, 1977.

HALLAUER, A.R.; SEARS, J.H. Changes in quantitative traits associated with inbreeding in a synthetic variety of maize. Crop Science, Madison, v.13, p.327-330, 1973.

LIMA, M.; MIRANDA FILHO, J.B.; GALLO, P.B. Inbreeding depression in Brazilian populations of maize (Zea mays L.). Maydica, Bergamo, v.29, p.203215, 1984.
NASS, L.L.; MIRANDA FILHO, J.B. Inbreeding depression rates of semi-exotic maize (Zea mays L.) populations. Revista Brasileira de Genética, Ribeirão Preto, v.18, p.585-592, 1995.

PACKER, D. Variabilidade genética e endogamia em quatro populações de milho (Zea mays L.). 1998. 102p. Tese (Doutorado)-Escola Superior de Agricultura "Luiz de Queiroz", Piracicaba, 1998.

RAMALHO, M.A.P.; FERREIRA, D.F.; OLIVEIRA, A.C. de. Experimentação em genética e melhoramento de plantas. 2.ed. Lavras: UFLA, 2005. 322p.

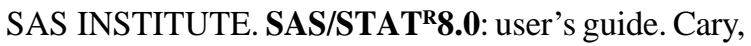
2000. 935p.

SOUZA SOBRINHO, F. Divergência genética de híbridos simples e alternativas para a obtenção de híbridos duplos de milho. 2001. 96p. Tese (Doutorado)Universidade Federal de Lavras, Lavras, 2001.

\section{TERESAWA JUNIOR, F. Seleção recorrente com} endogamia em duas populações de milho: avaliação quantitativa e perspectiva para seleção de híbridos. 1993. 169p. Dissertação (Mestrado)-Escola Superior de Agricultura “Luiz de Queiroz”, Piracicaba, 1993.

VASAL, S.K.; DHILLON, B.S.; SRINIVASAN, G.; MCLEAN, S.D.; ZHANG, S.H. Effect of S3 recurrent selection in four tropical maize populations on their selfed and randomly mated generations. Crop Science, Madison, v.35, p.697-702, 1995.

VENCOVSKY, R. Herança quantitativa. In: PATERNIANI, E.; VIÈGAS, G.P. (Eds.). Melhoramento e produção do milho no Brasil. 2.ed. Campinas: Fundação Cargill, 1987. p.122-201

VIANNA, R.T.; GAMA, E.E.G.; NASPOLINI FILHO, V.; MORO, J.R.; VENCOVSKY, R. Inbreeding depression of several introduced populations of maize (Zea mays L.). Maydica, Bergamo, v.27, p.151-157, 1982. 